\title{
AN INTERFEROMETRIC SURVEY OF MIRA VARIABLES AND
}

\section{SUPERGIANTS}

\author{
P.G. TUTHILL, C.A. HANIFF* and J.E. BALDWIN \\ MRAO, Cavendish Laboratory, \\ Madingley Road, Cambridge, \\ CBS OHA, UK
}

\begin{abstract}
We present preliminary results from an imaging survey of bright northern Mira variables and Supergiants. Diffraction-limited images of Mira exhibit the same elongation along PA $120^{\circ}$ as observed in previous observations. Of the other Mira variables observed, $R$ Cas is unambiguously detected as non-spherically symmetric. Our observations of $\alpha$-Her are consistent with there being a 'hot-spot' on its surface, similar to those we have previously detected on Betelgeuse.

Key words: Non-redundant-masking - Mira variables - supergiant stars - surface inhomogeneities.
\end{abstract}

\section{Introduction}

Following the success of our initial NRM experiments on Betelgeuse and Mira [1,2] we have begun a high-resolution imaging survey of all bright Mira variables and supergiants in the northern sky. Because of their extended atmospheres and red colours, these objects are ideally suited for interferometric study. Our primary aims include the detection of surface asymmetries and inhomogeneities, and the precise determination of angular diameters and limb darkenings. In combination with highprecision parallax measurements, these data will be useful diagnostics for dynamical atmospheric models of evolved stars. Our most recent observations were made using standard NRM and PRM techniques at the GHRIL facility on the William Herschel Telescope in January and July of 1992. Details of the experimental and data reduction procedures can be found elsewhere $[1,2]$.

\section{Survey Results}

Most of the objects observed (15/20) proved to be only partially resolved at $4 \mathrm{~m}$ baselines. Mapping has not been performed for these sources, although wavelength dependent diameter measurements were made. Results from a preliminary analysis of the data are summarized in table 1.

\section{Mira and Betelgeuse}

We have made periodic observations of these two sources since 1989, (representing 3 whole pulsation cycles for Mira) and find that they exhibit contrasting behavior. For Betelgeuse, the closure phase data are non-zero, indicating departures from spherical symmetry, and our reconstructed images show bright "hotspots" superimposed on the stellar disk. The hotspots have been observed to evolve on timescales of months and it will be necessary to observe at shorter intervals (of around a month) to examine this process in detail.

- also at Institute of Astronomy, Madingley Road, Cambridge, CB3 OHE, UK 
TABLE I

Best fit Uniform Disk Diameters of Survey Objects

\begin{tabular}{lccccl}
\hline $\begin{array}{c}\text { Object } \\
\text { name }\end{array}$ & $\begin{array}{c}\text { Type of } \\
\text { object }\end{array}$ & $\begin{array}{c}\text { Oscillation } \\
\text { phase }\end{array}$ & $\begin{array}{c}\text { Wavelength } \\
\text { (nm) }\end{array}$ & $\begin{array}{c}\text { Diameter } \\
\text { (mas) }\end{array}$ & Comments \\
\hline V Cvn & M III & .85 & 830 & $24 \pm 4$ & \\
R Cvn & M III & .94 & 830 & $\leq 20$ & unresolved \\
S Crb & M6 III & .19 & 830 & $22 \pm 6$ & \\
V Crb & C6,2 III & .88 & 830 & $21 \pm 2$ & \\
R Ser & M7 III & .73 & 700 & $\leq 20$ & unresolved \\
R Ser & M7 III & .73 & 830 & $\leq 20$ & unresolved \\
x Oph & M6.5 III & .73 & 830 & $\leq 30$ & \\
R Aql & M7 III & .06 & 700 & $25 \pm 4$ & \\
R Aql & M7 III & .06 & 710 & $30 \pm 4$ & \\
30 Her & M6 III & - & 700 & $\leq 20$ & unresolved \\
30 Her & M6 III & - & 710 & $20 \pm 3$ & \\
R Cyg & M III & .16 & 830 & $\leq 20$ & unresolved \\
R Aqr & M7 III & - & 830 & $23 \pm 6$ & \\
Chi Cyg & S6 III & .69 & 830 & $33 \pm 5$ & \\
Chi Cyg & S6 III & .69 & 905 & $33 \pm 3$ & \\
T Cep & M7 III & .20 & 700 & $34 \pm 1$ & \\
T Cep & M7 III & .20 & 905 & $28 \pm 8$ & \\
$\mu$ Cep & M2 I & - & 546 & $26 \pm 2$ & \\
$\mu$ Cep & M2 I & - & 633 & $25.5 \pm 2.5$ & \\
$\mu$ Cep & M2 I & - & 700 & $28.3 \pm 3$ & \\
$\mu$ Cep & M2 I & - & 710 & $26.6 \pm 3$ & \\
$\alpha$ Sco & M1-2 I & - & 700 & $33 \pm 3$ & \\
\hline & & & & & \\
\hline & & & & & \\
\end{tabular}

For Mira no such features are detectable, but the stellar disk is elliptically shaped. Our most recent images show the same position angle for the elongation ( $120^{\circ}$ ) as determined in January 1991 (see fig. 1), which suggests that this asymmetry is long-lived as compared to the variability timescale.

\section{Newly Mapped Sources}

Our most interesting results concern the imaging of fainter resolved objects not attempted on previous observing runs:

$\alpha$ Her (Fig. 2a) is a triple system, with an M5 giant primary. Images obtained at $633 \mathrm{~nm}$ and $710 \mathrm{~nm}$ both show an unresolved feature on the 40 mas stellar disk offset some 9 mas from the center along PA $-90^{\circ}$. This hot-spot contributes $\sim 7 \%$ of the stellar flux, and is probably similar to those seen on the surface of Betelgeuse.

Maps of the long period variable $\mathrm{R}$ Cas, obtained near maximum light, are strikingly elliptical (Fig. 2b), more so than Mira itself. Since this M7 giant has no close companion, the suggestion is that the preferred axis is intrinsic to the star, and not the result of any possible tidal interaction. 
These new results are surprising given the small number of sources observed in this preliminary sample, but may indicate a far higher occurrence of surface inhomogeneities than has previously been expected in these classes of stars.

\section{Acknowledgements}

JEB and CAH are grateful to the Royal Society for financial support.

\section{References}

[1] Buscher, D.F., Haniff, C.A., Baldwin, J.E., and Warner, P.J.: 1990, 'Detection of a bright feature on the surface of Betelgeuse', Mon. Not. R. Astr. Soc. 245, 7p.

[2] Wilson, R.W., Baldwin, J.E., Buscher, D.F., and Warner, P.J.: 1992, 'High resolution imaging of Betelgeuse and Mira', Mon. Not. R. Astr. Soc. 257, 369.

MRA IN $1991 \cdot 700$ NM

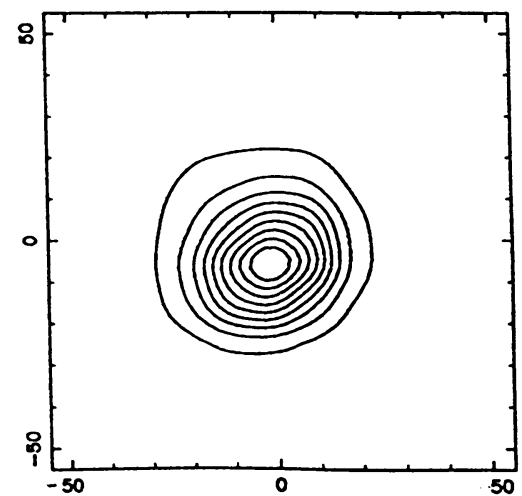

MIRA JUYY $1992 \odot 700 \mathrm{NM}$

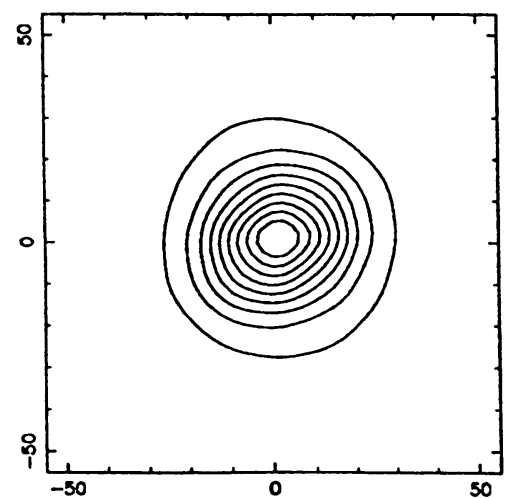

Fig. 1. Images of Mira at 700nm taken in Jan $1991(\phi=0.27)$ and Jul $1992(\phi=0.94)$. In this figure and fig. 2 North is to the bottom and East to the right, and the tickmarks are given in milliarcseconds. Contours are plotted from $10 \%$ to $90 \%$ of the peak flux at $10 \%$ intervals.

NPHA HER JULY $1992 \div$ G33NM

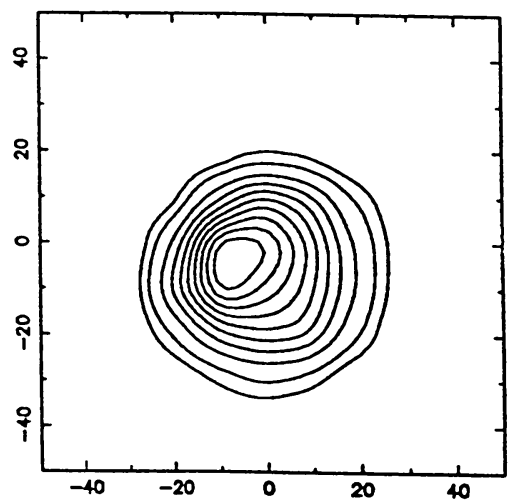

R CASS JUKY $1992 \bullet 700 \mathrm{NM}$

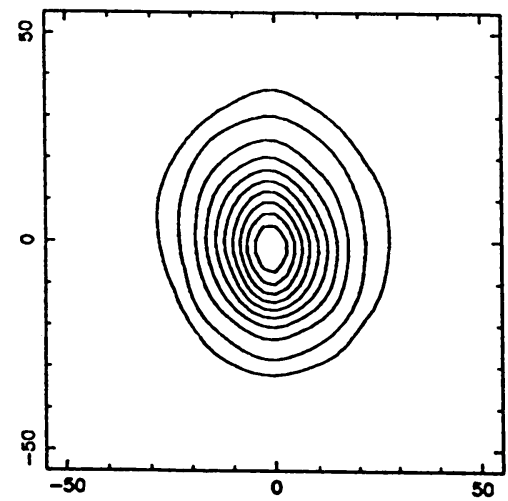

Fig. 2. Images of $\alpha$ Her (633nm) and R Cass (700nm) in Jul 1992. Contour levels are plotted at $5 \%, 10 \%, 20 \%, 30 \%, 40 \%, 50 \%, 60 \%, 70 \%, 80 \%$, and $90 \%$ of the peak flux. 